\title{
Immunogenicity and Protective Capacity of Schistosoma haematobium Recombinant cathepsin L Against Infection of Hamsters with S. haematobium
}

\author{
Abdel Aziz Nada ${ }^{1,2}$ (i) , Tallima Hatem ${ }^{1}(\mathbb{D})$, Abou El Dahab Marwa ${ }^{3}$, El Ridi Rashika ${ }^{1 *}$ (i) \\ ${ }^{1}$ Zoology Department, Faculty of Science, Cairo University, Giza 12613, Egypt. ${ }^{2}$ Chemistry Department, Faculty of Science, \\ Cairo University, Giza 12613, Egypt. ${ }^{3}$ Zoology Department, Faculty of Science, Ain Shams University, Cairo 11566, Egypt.
}

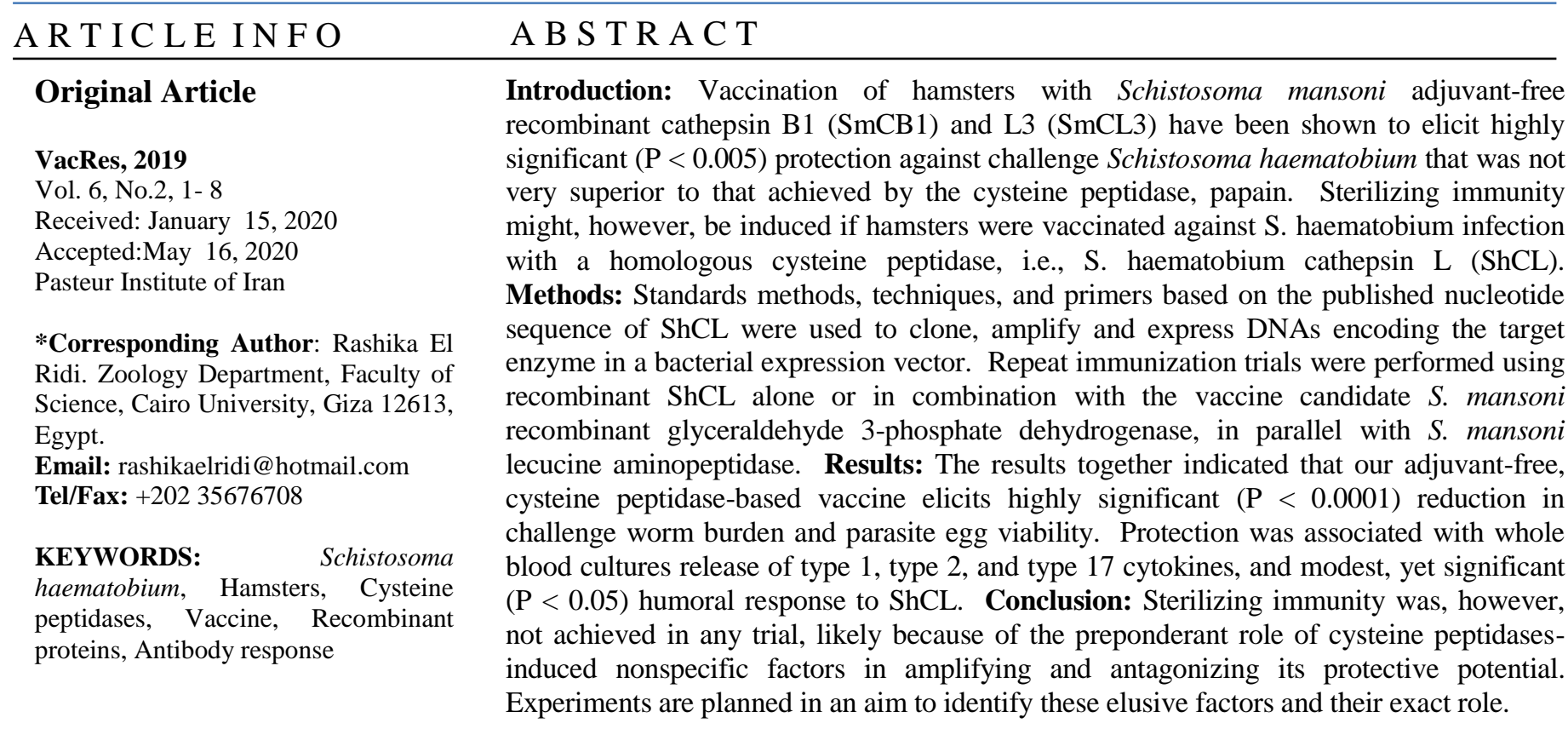

\section{Citation:}

Abdel Aziz N, Tallima H, Abou El Dahab M, El Ridi R. Immunogenicity and Protective Capacity of Schistosoma haematobium Recombinant cathepsin L Against Infection of Hamsters with S. haematobium. vacres. 2019; 6 (2):1-8

\section{INTRODUCTION}

Schistosomiasis is a parasitic disease, endemic in 74 countries of the developing world with around 500 million people, mostly children, infected and 800 million at risk of the infection. Human infections are essentially due to Schistosoma mansoni, Schistosoma haematobium, and Schistosoma japonicum. Schistosomiasis haematobium is prevalent only in the Middle East and Africa; however, it is more prevalent (54.4\%) than schistosomiasis mansoni $(40.8 \%)$, especially in Western and Southern sub-Saharan African countries [1]. Infection is initiated by cercariae penetrating host epidermis where they remain until their classical trilaminate membrane transforms into a double lipid layer. The larvae, now termed schistosomula, access the dermis and then penetrate into the dermal blood capillaries en route to the liver via the pulmonary vasculature. The worms develop and mature in the liver sinusoids, copulate, and then leave to their permanent abode in the mesenteric capillaries ( $S$. mansoni, S. japonicum) or vessels of the pelvic plexus (S. haematobium). Hundreds of eggs are deposited daily; about half of them exit via stools or urine to infect specific freshwater snails and continue the life cycle.
The rest are trapped into the host tissues, releasing antigens which initiate vigorous inflammatory immune responses that are responsible for the disease symptoms [1-3]. No commercial vaccine is available as yet.

The numerous molecules released, excreted and/or secreted (ESP) by the developing and migrating larvae are readily presented to the host lymphocytes, driving the induction of $\mathrm{T}$ helper (Th) effector cells and specific antibody-producing lymphocytes, and are accessible targets to the diverse immune effectors. Our Laboratory has identified many of the $S$. mansoni lung-stage larvae ESP, among which glyceraldehyde 3-phosphate dehydrogenase (SG3PDH), 14-3-3-like protein, 2Cys peroxiredoxin (PRX $=$ thioredoxin peroxidase, TPX), and calpain which are vaccine candidates are renowned for their immunogenicity and protective capacity [4]. We have proposed a mechanism of innate and acquired immunity-mediated schistosome attrition, whereby the larvae ESP are the "scent" which would allow the antibodies and immune cells to target, "hunt", pursue, and surround the migrating parasites. Interaction of antibodies and immune cells with ESP trigger the 
generation of inflammatory mediators and toxic radicals that may eventually lead to elimination of the crawling parasites [46]. This "chase" is certainly most effective in the narrow blood capillaries of the lung and the sinusoids of the liver, longknown to be the major sites of natural and immune elimination of schistosomes [4-6].

Accordingly, it was important to revise the major issue of defining the global cytokine profile conducive to effective schistosome larvae killing. We proposed a paradigm shift in vaccination against murine schistosomiasis and delineated the steps necessary for development of a sterilizing vaccine against schistosomiasis mansoni. We have provided evidence for our hypothesis stating that the vaccine formula for an effective schistosomiasis vaccine would use larval ESP in the context of a polarized type 2 , not type 1 , cytokine environment. Indeed immunizing outbred, akin to man, mice with recombinant SG3PDH (rSG3PDH) and TPX-derived peptides in a multiple antigen peptide (MAP) construct, in combination with the type 2-inducing papain, or the type 2 cytokines thymic stromal lymphopoietin (TSLP), interleukin (IL)-25, or IL-33 reproducibly and consistently elicited highly significant $(\mathrm{P}<$ 0.0001 ) $60-75 \%$ reduction in challenge worm burden and worm egg counts in liver and small intestine [7]. Our hypothesis was fully confirmed as immunization of outbred mice or hamsters with schistosome molecules, that are both ESP and type 2 immune responses-inducing, namely papain or the parasite cysteine peptidases, consistently and reproducibly elicited highly significant $(\mathrm{P}<0.0001)$ reduction (of 50-60\%) of challenge schistosome worm burden and worm egg load in liver and intestine as compared to unimmunized hosts [4, 8-13]. More specifically, hamsters immunized with $S$. mansoni cathepsin B1 (SmCB1) or L3 (SmCL3) displayed at day 10 post- challenge infection low antibody titers and weak but polarized cytokine type 2 responses to the immunogens. Both cysteine peptidases, alone or in combination, evoked reproducible and highly significant reduction in challenge worm burden $(>70 \%, \mathrm{P}<0.005)$ as well as significant reduction in worm egg counts and viability [13].

It is herein aimed to increase the protective potential of the adjuvant-free, cysteine peptidase-based vaccine against $S$. haematobium via cloning the homologous cathepsin $\mathrm{L}$, and examining its potential, alone or in combination with SG3PDH, in driving hamster immune responses towards the type 2 axis and ameliorating challenge $S$. haematobium infection sequelae.

\section{MATERIALS AND METHODS}

Cloning and Expression of S. haematobium Cathepsin L (ShCL)

Primer selection was based on published sequences for $S$. haematobium cathepsin L (gi|844841648; XM_012937844.1; XP_012793298.1 [14]. The mature product (648 bp) was targeted with forward primer [(5'-C ACC ATA CCA AAA AAC TTC GAT TG-3' (24 mer)], and reverse primer [(5'-CTA GTA GAT CAA CGC TGA C-3' (19 mer)], synthesized at Integrated DNA Technologies (IDT DNA, Leuven, Belgium) with additional CACC base pairs at the 5' of the forward primer, necessary for cloning in the selected expression vector (see below).

For cloning and amplification of cDNA coding for $S$. haematobium cathepsin L (ShCL) mature protein, RNA was extracted from 50 mixed-sex mature $S$. haematobium adult worms by using PureZOL (Bio-Rad, Hercules, CA, USA). Double stranded cDNA generation and amplification by RTPCR were performed using SuperScript III One-Step RT-PCR
System with Platinum ${ }^{\circledR}$ Taq High Fidelity kit (Thermo Fisher Scientific, Waltham, MA, USA), which produces blunt-end PCR products. Each sample $(10 \mu \mathrm{l})$ was analyzed by $1.5 \%$ agarose gel electrophoresis and ultraviolet (UV) trans illumination (Biometra, Göttingen, Germany) using standard procedures, described previously [4]. As the amplicon was of the expected size, the cDNA was isolated from the agarose band by Quantum Prep Freeze N Squeeze DNA Gel Extraction Spin Column (Bio-Rad). Purity and concentration of the purified product were assessed by agarose gel electrophoresis and UV trans illumination. DNA sequencing of the amplicon was performed at the University of Cape Town Core DNA Sequencing Service (Cape Town, South Africa).

The purified ShCL-coding cDNA was expressed in the Champion pET Directional TOPO Expression Kit ${ }^{\circledR}$ (InVitrogen; Carlsbad, CA, USA), following the manufacturer's instructions which required blunt-end PCR products containing CACC at the $5^{\prime}$ end, cloning into TOPO ${ }^{\circledR}$ vector, transformation and amplification in TOPO10 chemically competent Escherichia coli, preparation of plasmid, and then transformation and expression in the BL21 Star (DE3) chemically competent $E$. coli. The transformation reaction was grown in $200 \mathrm{~mL} \mathrm{LB}$ medium containing $100 \mu \mathrm{g} / \mathrm{ml}$ ampicillin (Sigma-Aldrich, St. Louis, MO, USA) until the OD600 reached 0.7 (mid-log phase), and incubated for $4 \mathrm{~h}$ at $37^{\circ} \mathrm{C}$ with shaking (300 rpm) in the presence of $1 \mathrm{mM}$ IPTG. The induced cells were sedimented, incubated in Dulbecco's phosphate-buffered saline, pH 7.1 (D-PBS) supplemented with $1 \mu \mathrm{g} / \mathrm{ml}$ leupeptin and $1 \mathrm{mM}$ PMSF as protease inhibitors, RNase T1 $(100 \mu \mathrm{g} / \mathrm{ml})$ and DNase $1(20 \mu \mathrm{g} / \mathrm{ml}$; all from Sigma-Aldrich) and sonicated (50 Watts for $2 \mathrm{~min}$ on ice). The suspension was then centrifuged at $10000 \mathrm{x} \mathrm{g}$ for $20 \mathrm{~min}$ and the soluble supernatant stored at $-76^{\circ} \mathrm{C}$. The sedimented bacteria were incubated for 15 min at room temperature in D-PBS containing $25 \%$ sucrose and $1 \%$ Triton-X100 (Bio-Rad), sonicated at 50 Watts for 2 min on ice and then centrifuged as above. The Triton soluble supernatant was stored at $-76^{\circ} \mathrm{C}$. Aliquots of uninduced and IPTG-induced bacteria and soluble and Triton soluble supernatants of cultures were analyzed by SDS-PAGE $(10 \%$ gel, reducing conditions).

The recombinant protein was purified from the soluble fraction by metal affinity chromatography using HiTrap Chelating HP $5 \mathrm{ml}$ columns (Amersham Biosciences AB, Uppsala, Sweden), following the manufacturer's instructions. The bound molecules were eluted and dialyzed overnight against $1 \mathrm{x}$ sterile D-PBS at $10^{\circ} \mathrm{C}$. Protein content in the purified fraction was measured by the Bradford assay and by spectrometry whereby the protein was measured at absorbance $280 \mathrm{~nm}$ (A280) and A260 (UV-Photometer GeneRay, Biometra) and the protein concentration was calculated by the formula: protein concentration $(\mathrm{mg} / \mathrm{ml})=(1.55 \mathrm{x} \mathrm{A280})-(0.76$ $x$ A260). Cathepsin L activity was determined by the fluorometric substrate assay as described [15-17]. Briefly, ShCL samples (10 and $50 \mu \mathrm{g}$ ) were incubated in duplicate wells of black 96 well plates (Greiner Bio-One, Kremsmünster, Austria), in parallel with 10 and $50 \mu \mathrm{g}$ of SmCL3 (courtesy of Dr. Jan Dvořák and Professor Dr. John P Dalton) in a total volume of $250 \mu \mathrm{l}$ of substrate/buffer mix (105.6 mM sodium mono phosphate, $14.4 \mathrm{mM}$ disodium phosphate, $1.2 \mathrm{mM}$ ethylenediamine tetraacetic ( $\mathrm{pH}$. 6.0), $2.5 \mathrm{mM}$ L-cysteine, $0.07 \% \quad(\mathrm{v} / \mathrm{v}) \quad$ Brij $35, \quad 0.006 \quad \mathrm{mM} \quad$ Z-Phe-ArgAMC(benzyloxycarbonyl-phenylalanylarginine- 7-amido-4methylcoumarin hydrochloride; Sigma) or Z-Arg-Arg-AMC substrate at $40 \mathrm{oC}$ for $30 \mathrm{~min}$. Release of free AMC was 
measured at excitation and emission wavelengths of 355 and $460 \mathrm{~nm}$, respectively, using Victor X4 Multilabel Plate Reader (PerkinElmer, Waltham, MA).

\section{Ethics Statement}

All animal experiments were performed following the recommendations of the current edition of the Guide for the Care and Use of Laboratory Animals, Institute of Laboratory Animal Resources, National Research Council, USA, and were approved by the Institutional Animal Care and Use Committee (IACUC) of the Faculty of Science, Cairo University, permit numbers CUFS F PHY 2114 and CUFS-F-Imm-5-15.

\section{Animals and Parasites}

Female Syrian hamsters (Mesocricetus auratus) were raised at the Schistosome Biological Materials Supply Program, Theodore Bilharz Research Institute (SBSP/TBRI), Giza, Egypt, until 6 week-old, and were maintained throughout experimentation at the animal facility of the Zoology Department, Faculty of Science, Cairo University. Cercariae of an Egyptian strain of $S$. haematobium were obtained from SBSP/TBRI, and used immediately after shedding from Bulinus truncatus snails. Hamsters were anesthetized, the abdomen shaved, wet with sterile deionized water, and then exposed to 100 cercariae in $100 \mu \mathrm{l}$ deionized water, protected from spilling by a sterile steel ring, as described [10, 12, 13].

\section{Serum Antibody Assays}

Blood samples were obtained from entirely naïve and from unimmunized and immunized hamsters ( 3 to 4 per group) 10 days (parasite lung-stage) following infection with $S$. haematobium cercariae. Sera were separated on an individual hamster basis, and stored at $-20^{\circ} \mathrm{C}$ until use. Hamster serum antibody titer and isotype were assessed by indirect enzymelinked immunosorbent assay (ELISA) for binding to 250 $\mathrm{ng} /$ well immunogen in duplicate wells as described $[10,12,13]$. Alkaline phosphatase (AKP)-labeled anti-hamster IgG $(\mathrm{H}+\mathrm{L})$ conjugate (Kirkegaard and Perry Laboratories, Gaithersburg, MD, USA) was diluted 1:1000. For each experiment, antibody isotypes of individual sera, diluted 1:50, was determined using biotin-labeled monoclonal antibodies to hamster IgG classes, IgG1 and IgG2 (Pharmingen, San Diego, CA, USA), and AKPlabeled streptavidin from Promega (Madison, WI, USA).

\section{Whole Blood Cultures}

Heparinized whole blood [18] $150 \mu \mathrm{l}$ samples were diluted 1:4 in RPMI-1640 medium, supplemented with 200 $\mathrm{U} / \mathrm{ml}$ penicillin, $200 \mu \mathrm{g} / \mathrm{ml}$ streptomycin, $25 \mathrm{mM}$ HEPES, and $20 \mu \mathrm{g} / \mathrm{ml}$ polymyxin B (Sigma-Aldrich) as an inhibitor of any lipopolysaccharide. Diluted blood samples were incubated in duplicate in wells of 48 well culture plates (Corning Costar, Corning, NY, USA) in the presence of 0 or $10 \mu \mathrm{g}$ membrane $(45 \mu \mathrm{m})$-filtered ShCL for $72 \mathrm{~h}$ at $37^{\circ} \mathrm{C} / 3 \% \mathrm{CO} 2$. Cultures were then centrifuged at $400 \mathrm{x}$ g for $10 \mathrm{~min}$ and the cell-free supernatants transferred into wells of sterile plate, and stored at $-76^{\circ} \mathrm{C}$ until assayed for cytokine release.

\section{Cytokine Assays}

Serum and culture supernatants were assayed by capture ELISA in duplicate for levels of released hamster IL-4, IL-5, IL-13, IL-17, and IFN- $\gamma$ (MyBioSource, Inc., San Diego, CA, USA) following the manufacturer's instructions.

\section{Parasitological Parameters}

Worm burden as well as liver and intestine worm egg load in individual hamsters ( 5 to 8 per group) were evaluated 12 weeks post infection with viable $S$. haematobium cercariae. Percent change was evaluated by the formula: $\%$ change = [mean number in infected controls - mean number in infected, treated hamsters / mean number in infected controls] $\times 100$. Percentages of egg developmental stages were evaluated using 3-5 fragments of the ileum and the large intestine. After washing in saline solution and slight drying on absorbent paper, each intestinal fragment was placed between 2 slides and analyzed by light microscopy to classify the eggs. For each fragment, up to 100 eggs were counted and classified according to their developmental stage as immature, viable eggs; mature, viable eggs; and non-viable calcified eggs. Liver sections from each control and test hamster were stained with haematoxylin and eosin and examined for the number and diameter of granulomas surrounding eggs $[12,13]$.

\section{Experimental Design}

For Experiment 1, a total of 20 female Syrian hamsters were randomly distributed into two groups of 10 hamsters. Hamsters were subcutaneously injected at the tail base region, twice, with a 3 week-interval, with $200 \mu \mathrm{l}$ D-PBS containing 0 (control group), or $20 \mu \mathrm{g} \mathrm{S}$. haematobium cathepsin L (ShCL). Eight weeks after the second immunization, the control and immunized hamsters were percutaneously exposed to 100 cercariae of S. haematobium, and parasitological parameters were evaluated 12 weeks post challenge infection, as described $[10,12,13]$.

For Experiment 2, 3 of 41 female Syrian hamsters were left unimmunized and uninfected and considered naïve animals. A total of 38 hamsters were randomly assigned to 3 groups of 10 hamsters each and a group of 8 hosts. Hamsters were subcutaneously injected at the tail base region, twice, with a 3week-interval, with $200 \mu \mathrm{l}$ D-PBS containing 0 (control group), $10 \mu \mathrm{g}$ recombinant (r) ShCL, $5 \mu \mathrm{g}$ ShCL plus $5 \mu \mathrm{g}$ rSG3PDH (10 hamsters per group) and 8 hamsters with $10 \mu \mathrm{g}$ rleucine aminopeptidase (a gift of Professor Dr. John Dalton). Five weeks after the second immunization, the control and immunized hamsters were percutaneously exposed to 100 cercariae of $S$. haematobium. On day 10 , when a large proportion of migrating larvae are in the lung capillaries, serum and whole blood cultures supernatants from each of 2-3 hamsters/group were assessed for immunological responses to the immunogen. Parasitological parameters were evaluated for 5-8 hamsters per group, 12 weeks post challenge infection [10, $12,13]$.

\section{Statistical Analyses}

All values were tested for normality. ANOVA, MannWhitney or Student's t 2-tailed test were used to analyze the statistical significance of differences between experimental and control values and considered significant at $\mathrm{P}<0.05$ (GraphPad InStat, San Diego, CA, USA).

\section{RESULTS}

\section{ShCL Gene Cloning and Expression}

The product obtained following gene cloning and amplification was analyzed by $1.5 \%$ agarose gel electrophoresis, and was of the expected size (648 bp) as shown before (Fig. 1A) and after (Fig. 1B) purification. DNA sequencing, translation, and blasting of the deduced amino acid 
sequencing revealed $98 \%$ homology of the amplicon with $S$. haematobium cathepsin $\mathrm{L}$ at amino acid level (Fig. 2A, B). The induced product was found essentially in the soluble but also in the Triton soluble compartments around $30 \mathrm{kDa}$ (Fig. 3). The recombinant protein was purified from the soluble fraction by metal affinity chromatography. The bound molecules were eluted and then dialyzed overnight against $1 \mathrm{x}$ sterile D-PBS. Purity and molecular mass of $2.0 \mu \mathrm{g}$ of the purified product is shown in Fig. 4.

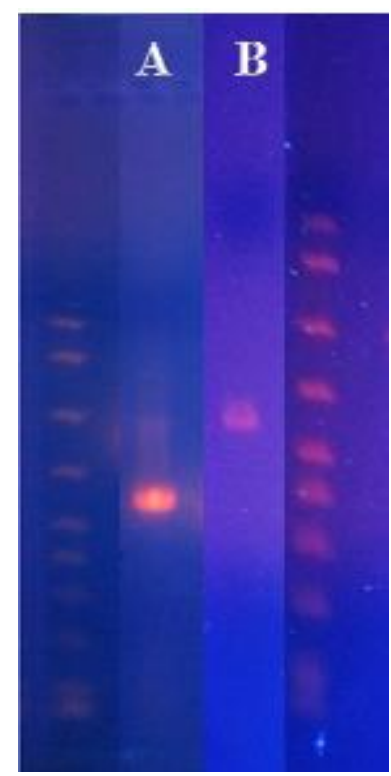

Fig. 1. Amplicon cDNA profile. Agarose gel electrophoresis was used to examine amplicon cDNA size and purity before (A) and after (B) purification, in parallel with DNA AmpliSize Molecular Ruler of BioRad, from top 2000, 1500, 1000, 700, 500, 400, 300, 200, 100, 50 bp. The mature ShCL cDNA is seen between 500 and 700 bp marks (640 bp).
CGGCCKCTACTGAGTAAAAATCAAGGAATGTGTG GCTCTTGTTGGGCGTTCTCAACCACTGGTAATGTT GAGAGTCAGTGGTTCCGCAAAACTGGAAAGTTAT TATCATTAAGTGAGCAGCAACTTGTTGACTGYGA CGGTTTAGATGATGGTTGTAATGGTGGTCTTCCAT CGAATGCCTATGAATCAATCATAAAAATGGGTGG TTTAATGCTTGAAGATAATTATCCTTATGATGCTA AAAATGAGAAATGTCATTTGAAAGCTGATRATGT TGCAGCTTACATTAATAGTTCAGTAAATTTAACTC AAGATGAAACAGAACTTGCTGCATGGCTTTATCA TAATTCARCGATTAGTGTTGGTATGAATGCGWTG CTTTTACAGTTTTATCGACATGGAATTAGTCATCC ATGGTGGATCTTCTGTTCAAAGTATCTGCTTGATC ATGCGGTTTTACTTGTTGGCTATGGAGTTTCCGAA RAAAATGAGCCMTTCTGGATTGTAAAAAACAGTT GGGGTGTTGAATGGGGTGAAAAAGGTTACTTCCG TGTGTATCGAGGTGATGGTACTTGTGGCATTAATA CTGTTGCTACGTCAGCGTTAWTYCTACTAGA

Fig. 2. (A). The Amplicon DNA sequence; 617 bp.

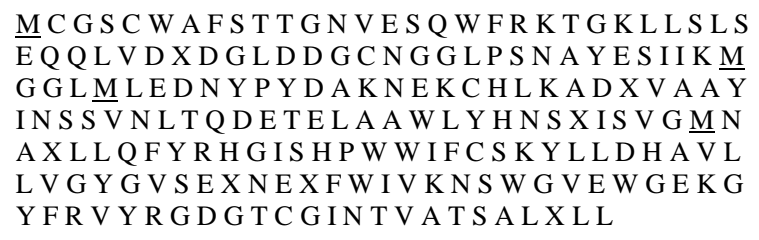
EQQLVDXDGLD D GCNGGLPS N A Y E S I K K $\underline{M}$ G G L M LE D N Y P Y D A KNEKCHLKA D X V A A Y IN S S V N L T Q DET E L A A W L Y HNS XIS V G M N AXLLQFYRHGISHPWWIFCSKYLLDHAVL LV G Y GVS EXNEXFW IV KN S W GVEW GEK G YFR V Y R GD GT C GINTVATS A L X L L

Fig. 2. (B) The amplicon cDNA translation. Using Expasy translate tool, the amplicon sequence yielded a polypeptide of 195 amino acids, which displayed $98 \%$ homology with S. haematobium cathepsin L (XP_012793298.1).

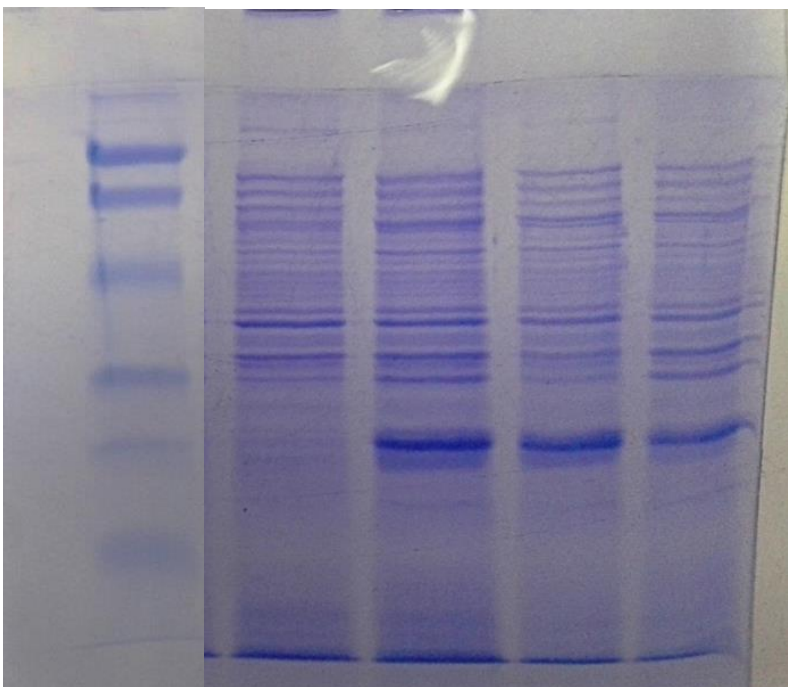

Fig. 3. Amplicon expression profile. Samples of uninduced (lane 1) IPTG-induced (lane 2), D-PBS-soluble (lane 3), and Triton-soluble (lane 4) bacterial molecules were separated by SDS-PAGE, and stained with Coomassie Blue. On left (lane M) prestained protein molecular mass markers of 20.486, 29.059, 36.811, 50.443, 84.174 and $105.203 \mathrm{kDa}$ (Bio-Rad). The arrow points to the induced mature ShCL product, of around $30 \mathrm{kDa}$, present in D-PBS-soluble and Triton-soluble supernatants.

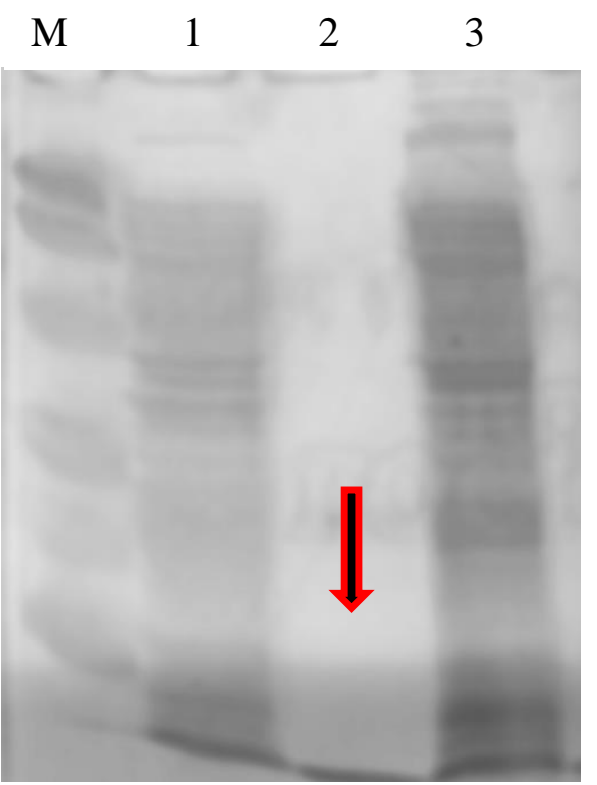

Fig. 4. Purified recombinant product profile. Samples of uninduced (lane 1), IPTG-induced (lane 3) soluble supernatants of recombinant bacteria, and the nickel affinity column-purified product molecules (lane 2) were separated by SDS-PAGE, and stained with Coomassie Blue. On left (lane M), prestained protein molecular mass markers of 20.486, 29.059, 36.811, 50.443, 84.174 and $105.203 \mathrm{kDa}$ (Bio-Rad). The arrow points to the purified mature ShCL product, of around $30 \mathrm{kDa}$. 
Of note, duplicate experiments indicated that ShCL displays approximately $10 \%$ of the activity shown by SmCL3 in fluorometric substrate assay for Cathepsin L activity [17].

Effects of Immunization with Recombinant ShCL on the Parasite Burden

Recombinant ShCL elicited highly significant $(\mathrm{P}<$ 0.0001 ) reduction of $52.3 \%$ in total worm burden (Fig. 5). Immunization with ShCL did not greatly reduce the number of eggs in the liver or large intestine (Fig. 5); yet, was associated with significant $(\mathrm{P}<0.02)$ decrease in the percent of mature ova, and highly significant $(\mathrm{P}=0.0002)$ increase in the percentage of dead ova that reached the large intestine (Table 1). Most importantly, immunization with ShCL resulted into significant $(\mathrm{P}<0.02)$ decrease in the number and highly significant $(\mathrm{P}<0.0001)$ reduction in the diameter of granulomas (Table 2), in support of the oogram information on the low viability of parasite eggs in the vaccinated group (Table $1)$.
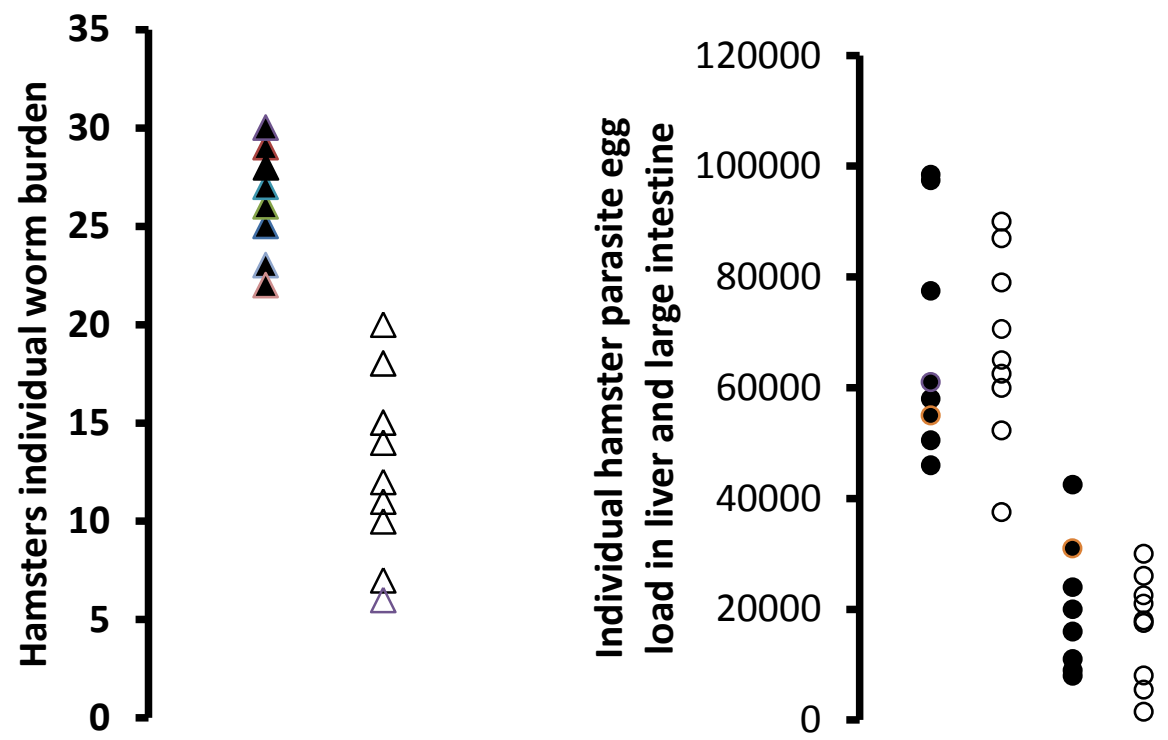

\begin{tabular}{|lcc|cc|cc|}
\hline & & & \multicolumn{2}{|c|}{ Liver } & \multicolumn{2}{c|}{ Large intestine } \\
\hline Mean & 26.2 & 12.5 & 68000 & 67094 & 20187 & 16666 \\
\hline SD & 2.8 & 4.5 & 20700 & 16759 & 11982 & 9682 \\
\hline $\boldsymbol{P}$ value & & $<0.0001$ & \multicolumn{2}{|c|}{ NS } & \multicolumn{2}{c|}{ NS } \\
\hline
\end{tabular}

Fig. 5. Effect of immunization with recombinant mature ShCL on challenge worm and egg burden. Each point represents values in individual unimmunized (black fill) and ShCL-immunized (no fill) hamsters. Significance of differences between vaccinated versus infection controls was assessed by Student's-"t"- and Mann-Whitney tests. NS = not significant.

Table 1. Effect of immunization with ShCL on egg development of challenge S. haematobium infection in hamsters*.

\begin{tabular}{|c|c|c|c|c|c|c|}
\hline \multirow{2}{*}{ Animal group } & & \multicolumn{4}{|c|}{ Mean \% ova developmental stages \pm SE } & \\
\cline { 2 - 7 } & Immature & P & Mature & P & Dead & P \\
\hline Controls & $22.18 \pm 8.20$ & & $65.72 \pm 11.66$ & & $12.06 \pm 4.05$ & \\
\hline ShCL & $21.68 \pm 4.24$ & NS & $31.38 \pm 6.67$ & 0.033 & $46.68 \pm 3.26$ & 0.0002 \\
\hline
\end{tabular}

* Hamsters were vaccinated with ShCL, challenged 8 weeks after second immunization with 100 cercariae of S. haematobium, and assessed for parasitological parameters twelve weeks post infection whereby 10 fragments of large intestine were examined. A minimum of 300 eggs were counted for each animal. Significance of differences of percentages of different developmental stages in large intestine of vaccinated versus infection controls was assessed by Student's-"t"- test. NS = not significant.

Table 2. Effect of immunization with ShCL on liver granulomas induced by challenge S. haematobium in hamsters*

\begin{tabular}{|l|l|l|}
\hline \multicolumn{1}{|c|}{ Granulomas } & Controls & ShCL \\
\hline Number & & \\
Mean \pm SD & $83.1 \pm 39.4$ & $44.2 \pm 19.3$ \\
$P$ value & & 0.018 \\
Reduction \% & & 46.8 \\
\hline Diameter & $329.2 \pm 52.1$ & $169.4 \pm 34.8$ \\
Mean \pm SD & & $<0.0001$ \\
$P$ value & & 48.5 \\
Reduction \% & & \\
\hline
\end{tabular}

*Hamsters were vaccinated with ShCL, challenged 8 weeks after second immunization with 100 cercariae of S. haematobium, and assessed for number and diameter of granulomas in liver. The data show mean + SD of granuloma parameters in 10 fields (x100) of 3 slides for each control and vaccinated hamster. NS $=$ not significant, as assessed by the Student's t- test (two-tailed $\mathrm{P}$ value). Reduction $\%=$ mean number in unimmunized hamsters - mean number in cysteine peptidase-immunized hamsters/ mean number in unimmunized hamsters x 100 . 


\section{Cytokines Responses to Recombinant ShCL}

Whole blood cultures from 3 naïve unimmunized and uninfected hamsters failed to produce detectable levels of IL-4, IL-5, IL-13, IL-17 or IFN- $\gamma$. The first and second order cytokine responses to $S$. haematobium were reflected, respectively in unstimulated and ShCL-stimulated whole blood cultures tested 10 days after infection, and involved release of significant amounts of IL-13 and IL-17. Whole blood cultures of ShCL-immunized hamsters expressed memory responses to the immunogen as judged by release of IL-13 and IFN- $\gamma$. The memory response to ShCL of ShCL + SG3PDH-immunized hamsters was evidenced by release of significantly $(\mathrm{P}<0.05)$ higher levels of IL-4, IL-13, IL-17, and IFN- $\gamma$ as compared to unstimulated cultures

Ten day infected hamsters did not produce detectable levels of anti-ShCL antibodies (data not shown).
Effects of Immunization with Recombinant ShCL alone or with SG3PDH

Immunization with ShCL alone or in conjunction with SG3PDH elicited increase in hamster serum antibody binding to ShCL (Fig. 6), involving essentially the IgG2 isotype. Regarding parasitological findings, ShCL alone or in combination with SG3PDH elicited highly significant $(\mathrm{P}=$ 0.0007 and $\mathrm{P}=0.0003$ ) reduction in total, male, and female worm burden, with highest decrease (65\%; P (Mann-Whitney) $=0.0003)$ achieved in hamsters immunized with ShCL + SG3PDH. The highly significant $(\mathrm{P}<0.001)$ reduction in $S$. haematobium worm numbers achieved following immunization with the cysteine peptidase-based vaccine is emphasized by the modest protective capacity $(\mathrm{P}<0.05 ; 31 \%)$ of leucine amino peptidase (leucine AP) assayed in a parallel hamsters group (Table3).

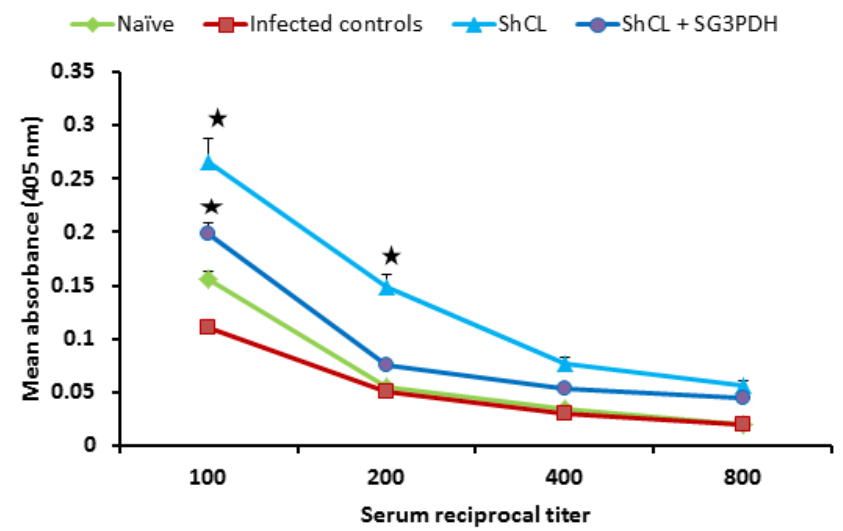

Fig. 6. Hamster serum antibody response to ShCL. Each point represents mean + SD absorbance of sera from 3 to 4 hamsters per group, assayed in quadruplicates by ELISA. Asterisks indicate significant $(\mathrm{P}<0.05$, ANOVA and " $\mathrm{t}$ " test $)$ ) differences from unimmunized infected controls.

Table 3. Effect of immunization with ShCL alone or in combination with rSG3PDH on parasitological parameters of challenge S. haematobium infection in hamsters*.

\begin{tabular}{|c|c|c|c|c|}
\hline \multirow{2}{*}{ PARAMETER } & \multicolumn{4}{|c|}{ VACCINE } \\
\hline & Infected controls & ShCL & ShCL+SG3PDH & Leucine AP \\
\hline $\begin{array}{l}\text { Total worm burden } \\
\text { Mean } \pm \mathrm{SD} \\
P \text { value } \\
\text { Reduction } \%\end{array}$ & $33.7 \pm 9.0$ & $\begin{array}{l}13.5 \pm 2.1 \\
0.0007 \\
60.0\end{array}$ & $\begin{array}{l}11.7 \pm 4.5 \\
0.0003 \\
65.2\end{array}$ & $\begin{array}{l}23.2 \pm 3.8 \\
0.0325 \\
31.1\end{array}$ \\
\hline $\begin{array}{l}\text { Male worm burden } \\
\text { Mean } \pm \text { SD } \\
\text { P value } \\
\text { Reduction } \%\end{array}$ & $17.2 \pm 5.0$ & $\begin{array}{l}6.6 \pm 1.3 \\
0.0007 \\
61.6\end{array}$ & $\begin{array}{l}5.5 \pm 1.9 \\
0.0003 \\
68.0\end{array}$ & $\begin{array}{l}11.6 \pm 2.3 \\
0.0397 \\
32.5\end{array}$ \\
\hline $\begin{array}{l}\text { Female worm burden } \\
\text { Mean } \pm \text { SD } \\
\text { P value } \\
\text { Reduction } \%\end{array}$ & $16.5 \pm 4.2$ & $\begin{array}{l}6.8 \pm 1.4 \\
0.0007 \\
58.7\end{array}$ & $\begin{array}{l}6.1 \pm 2.8 \\
0.0003 \\
63.0\end{array}$ & $\begin{array}{l}11.6 \pm 1.9 \\
0.0342 \\
29.6\end{array}$ \\
\hline $\begin{array}{l}\text { Liver egg counts } \\
\text { Mean } \pm \text { SD } \\
P \text { value } \\
\text { Reduction } \%\end{array}$ & $37875 \pm 8412$ & $\begin{array}{l}27083 \pm 6304 \\
0.021 \\
28.5\end{array}$ & $\begin{array}{l}42357 \pm 8385 \\
\text { NS }\end{array}$ & $\begin{array}{l}23500 \pm 5024 \\
0.0101 \\
33.5\end{array}$ \\
\hline $\begin{array}{l}\text { Large int. egg counts } \\
\text { Mean } \pm \text { SD } \\
P \text { value } \\
\text { Reduction } \%\end{array}$ & $8500 \pm 6595$ & $\begin{array}{l}9750 \pm 2824 \\
\text { NS }\end{array}$ & $\begin{array}{l}2857 \pm 1435 \\
0.048 \\
66.3\end{array}$ & $\begin{array}{l}2200 \pm 1303 \\
0.033 \\
74.1\end{array}$ \\
\hline
\end{tabular}

*Hamsters were vaccinated with ShCL alone or in combination with SG3PDH or with control antigen, leucine aminopeptidase (Leucine AP), challenged 5 weeks after second immunization with 100 cercariae of $S$. haematobium, and assessed for parasitological parameters twelve weeks post infection. Reduction $\%=$ mean number in unimmunized hamsters - mean number in immunized hamsters/mean number in unimmunized hamsters x 100. Data were statistically analyzed by ANOVA and the Mann-Whitney tests (two-tailed $P$ value). 
Table 4. Effect of immunization with ShCL alone or in combination with SG3PDH on development of challenge S. haematobium eggs in hamsters*.

\begin{tabular}{|c|c|c|c|c|c|c|}
\hline Animal group & & & Mean \% egg developmental stages \pm SE & & & \\
& & & & & \\
& immature ova & P-value & Mature ova & P-value & Dead ova & P-value (Change\%) \\
\hline Control & $53.49 \pm 21.11$ & & $49.64 \pm 19.35$ & & $9.04 \pm 5.24$ & \\
\hline ShCL & $24.75 \pm 24.09$ & NS & $51.26 \pm 23.36$ & NS & $28.94 \pm 19.08$ & $0.023(68 \%)$ \\
\hline $\begin{array}{c}\text { ShCL }+ \\
\text { SG3PDH }\end{array}$ & $27.90 \pm 10.84$ & NS & $15.67 \pm 5.75$ & $0.02 *$ & $60.85 \pm 12.87$ & $0.0001(85 \%)$ \\
\hline Leucine AP & $60.83 \pm 13.16$ & NS & $42.88 \pm 31.08$ & NS & $10.00 \pm 5.23$ & \\
\hline
\end{tabular}

*Five weeks after vaccination, control and immunized hamsters were challenged with 100 cercariae of S. haematobium, and assessed for parasitological parameters twelve weeks post infection whereby 10 fragments of large intestine were examined. A minimum of 300 eggs were counted for each animal. Leucine AP $=$ leucine aminopeptidase. Significance of differences of percentages of different developmental stages in large intestine of vaccinated versus infection controls was assessed by Student's "t"- test. NS = not significant.

The experimental data revealed that our cysteine peptidasebased vaccine does not elicit considerable decrease in challenge worm egg deposition (Table 3), but in sharp contrast to leucine amino peptidase effect, the great majority of the produced eggs are dead, especially if SG3PDH is included in the vaccine formula (Table 4). In support, significant $(\mathrm{P}<0.05)$ decrease in egg counts was observed in large intestine of ShCL + SG3PDH-immunized hamsters (Table 3).

\section{DISCUSSION}

Despite that hamster immunization with $S$. mansoni cysteine peptidases elicited reduction in challenge $S$. haematobium burden that reached $>70 \%$, we thought that sterilizing immunity would be induced if we vaccinated hamsters with a homologous cysteine peptidase, i.e., $S$. haematobium cathepsin L (ShCL). Accordingly, standards methods and techniques and primers based on the published nucleotide sequence of ShCL allowed us to successfully clone and amplify cDNA encoding the target enzyme, which displayed $98 \%$ amino acid homology with $S$. haematobium cathepsin L. Expression in a bacterial vector resulted in production of a $30 \mathrm{kDa}$ molecule that lacked distinct peptidase activity. None of 3 recombinant $S$. mansoni cathepsins B and L which had been previously expressed in E. coli exhibited detectable proteolytic activity. Absence of activity was expected as these enzymes and our recombinant protein possess many cysteine residues which have difficulty in refolding within the reducing milieu of the E. coli system in order to form 6 disulphide bonds [17, 19-21]. Expression in yeast is recommended for helminth derived cysteine peptidases [8-10, 17, 19-21]. Despite this lack of distinct proteolytic activity, ShCL induced a highly significant $(\mathrm{P}<0.0001$, Mann-Whitney test) reduction of 50-60\% in S. haematobium challenge worm burden in hamsters, in the range of that of the enzymatically active SmCB1 and SmCL3 [13]. This result is in accord with our proposal that both active and inactive cysteine peptidases possess anti-schistosomiasis protective potential [22]. It is remarkable that SmCL3 (ABV71063.1) [17] consistently displayed protective potential against challenge $S$. haematobium infection in hamsters [13] despite showing only 39\% identities and $56 \%$ positives with ShCL. S. mansoni cathepsin B1 (CAD44624.1) [21] was even more protective than SmCL3 [13] and ShCLlin that model, while showing with ShCL $26 \%$ identities and $41 \%$ positives. The data together support our suggestion that the cysteine peptidase vaccine protective activity is essentially due to shared conformational or linear motifs, epitopes, determinants in the cysteine peptidases polypeptides, in addition to, or independently of their enzymatic activity [22].

SmCB1, SmCL3 [13] or ShCL all failed to elicit decrease in liver egg load and highly significant reduction in large intestine egg count. Yet, the induced innate and/or acquired immunity led to considerable impairment of egg development and viability, resulting in the highly significant decrease $(\mathrm{P}<$ 0.0001 ) in size of liver granulomas. Of note, hamster immunization with $S$. mansoni leucine amino peptidase led to a modest decrease in challenge $S$. haematobium worm burden, and entirely failed to alter egg development and viability.

The mechanism of the cysteine peptidases antischistosomiasis protective mechanism appears to not depend on the activity of specific antibodies at the lung-stage, likely because memory responses would not be elicited before the time the worms grow, begin to eat and release the enzymes, at the liver stage [23]. Nevertheless, the immune responses results confirmed the poor immunogenicity of the cysteine peptidases of the L family and their ability to elicit type 1, type 2 , and type 17 cytokines [8-10, 13, 17].

Addition of SG3PDH consistently led to increase in the protective potential of our cysteine peptidase-based vaccine [712]. The reason perhaps resides in the fact that this enzyme is released by schistosomes larvae immediately upon infection, as well as, developing, and adult worms, inducing memory responses at the lung- and liver stages [8, 11]. Attrition of worms at the lung- and liver stage $[5,6,24]$ explains the higher reduction in worm burden and egg load in large intestine and the increase in percent of dead ova in intestine upon including SG3PDH in the vaccine formula. Immune responses elicited by ShCL alone or combined with SG3PDH were obviously limited, perhaps explaining why sterilizing immunity was not achieved. Additionally, it is possible that, contrary to leucine amino peptidase, our cysteine-based vaccine elicited predominant type 2 immune responses that resulted into generation of non-specific defense factors, which promote, yet also antagonize the cysteine peptidases-mediated schistosomicidal and ovocidal effects [22, 25].

\section{ACKNOWLEDGEMENT}

Thanks are due to Professor John Dalton (Queen University Belfast, Northern Ireland, UK) for the gift of S. mansoni recombinant leucine aminopeptidase and cathepsin L3. The 
research was funded by the Science and Technology Development Fund (STDF) grant ID. 13874 to R. El Ridi and H. Tallima.

\section{CONFLICT OF INTEREST}

The authors declare they have no conflict of interests.

\section{REFERENCES}

1. McManus DP, Dunne DW, Sacko M, Utzinger J, Vennervald BJ, Zhou XN. Schistosomiasis. Nat Rev Dis Primers. 2018;4(1):13. doi: 10.1038/s41572-018-0013-8.

2. Barsoum RS. Urinary schistosomiasis: review. J Adv Res. 2013;4(5):453-9. doi: 10.1016/j.jare.2012.08.004.

3. Barsoum RS, Esmat G, El-Baz T. Human schistosomiasis: clinical perspective: review. J Adv Res. 2013;4(5):433-44. doi: 10.1016/j.jare.2013.01.005.

4. El Ridi R, Tallima H. Schistosoma mansoni ex vivo lung-stage larvae excretory-secretory antigens as vaccine candidates against schistosomiasis. Vaccine. 2009; 27(5):666-73. doi: 10.1016/j.vaccine.2008.11.039.

5 El Ridi RA, Tallima HA. Novel therapeutic and prevention approaches for schistosomiasis: review. J Adv Res. 2013;4(5):467-78. doi: 10.1016/j.jare.2012.05.002.

6. El Ridi R, Tallima H, Migliardo F. Biochemical and biophysical methodologies open the road for effective schistosomiasis therapy and vaccination. Biochim Biophys Acta Gen Subj. 2017;1861(1 Pt B):36133620. doi: 10.1016/j.bbagen.2016.03.036.

7. El Ridi R, Tallima H. Vaccine-induced protection against murine schistosomiasis mansoni with larval excretory-secretory antigens and papain or type-2 cytokines. J Parasitol. 2013;99(2):194-202. doi: 10.1645/GE3186.1 .

8. El Ridi R, Tallima H, Selim S, Donnelly S, Cotton S, Gonzales Santana B, Dalton JP. Cysteine peptidases as schistosomiasis vaccines with inbuilt adjuvanticity. PLoS One. 2014;9(1):e85401. doi: 10.1371/journal.pone.0085401.

9. El Ridi R, Tallima H, Dalton JP, Donnelly S. Induction of protective immune responses against schistosomiasis using functionally active cysteine peptidases. Front Genet. 2014;5:119. doi: 10.3389/fgene.2014.00119. 10. Tallima H, Dalton JP, El Ridi R. Induction of protective immune responses against schistosomiasis haematobium in hamsters and mice using cysteine peptidase-based vaccine. Front Immunol. 2015;6:130. doi: 10.3389/fimmu.2015.00130.

11. Abdel Aziz N, Tallima H, Hafez EA, El Ridi R. Papain-based vaccination modulates Schistosoma mansoni infection-induced cytokine signals. Scand J Immunol. 2016;83(2):128-38. doi: 10.1111/sji.12399.

12. Tallima H, Dvořák J, Kareem S, Abou El Dahab M, Abdel Aziz N, Dalton JP, El Ridi R. Protective immune responses against Schistosoma mansoni infection by immunization with functionally active gut-derived cysteine peptidases alone and in combination with glyceraldehyde 3phosphate dehydrogenase. PLoS Negl Trop Dis. 2017;11(3):e0005443. doi: 10.1371/journal.pntd.0005443.

13. Tallima H, Abou El Dahab M, Kareem S, Dalton JP, El Ridi R. Protection against Schistosoma haematobium infection in hamsters by immunization with Schistosoma mansoni gut-derived cysteine peptidases, SmCB1 and SmCL3. Vaccine. 2017;35(50):6977-6983. doi 10.1016/j.vaccine.2017.10.069.

14. Stroehlein AJ, Korhonen PK, Chong TM, Lim YL, Chan KG, Webster $\mathrm{B}$, et al. High-quality Schistosoma haematobium genome achieved by single-molecule and long-range sequencing. Gigascience. 2019;8(9). pii: giz108. doi: 10.1093/gigascience/giz108.

15. Kuhelj R, Dolinar M, Pungercar J, Turk V. The preparation of catalytically active human cathepsin B from its precursor expressed in Escherichia coli in the form of inclusion bodies. Eur $\mathrm{J}$ Biochem. 1995;229(2):533-9.

16. Collins PR, Stack CM, O'Neill SM, Doyle S, Ryan T, Brennan GP, et al. Cathepsin L1, the major protease involved in liver fluke (Fasciola hepatica) virulence: propeptide cleavage sites and autoactivation of the zymogen secreted from gastrodermal cells. J Biol Chem. 2004; 279(17):17038-46.

17. Dvorák J, Mashiyama ST, Sajid M, Braschi S, Delcroix M, Schneider EL, et al. SmCL3, a gastrodermal cysteine protease of the human blood fluke Schistosoma mansoni. PLoS Negl Trop Dis 2009;3(6):e449. doi: 10.1371/journal.pntd.0000449.

18. Ai W, Li H, Song N, Li L, Chen H. Optimal method to stimulate cytokine production and its use in immunotoxicity assessment. Int $\mathrm{J}$ Environ Res Public Health. 2013;10(9):3834-42. doi 10.3390/ijerph10093834.

19. Dolinar M, Maganja DB, Turk V. Expression of full-length human procathepsin L cDNA in Escherichia coli and refolding of the expression product. Biol Chem Hoppe Seyler. 1995;376(6):385-8.

20. Dalton JP, Clough KA, Jones MK, Brindley PJ. Characterization of the cathepsin-like cysteine proteinases of Schistosoma mansoni. Infect Immun. 1996;64(4):1328-34.

21. Sajid M, McKerrow JH, Hansell E, Mathieu MA, Lucas KD, Hsieh I, et al. Functional expression and characterization of Schistosoma mansoni cathepsin $\mathrm{B}$ and its trans-activation by an endogenous asparaginyl endopeptidase. Mol Biochem Parasitol 2003 ;131(1):65-75.

22. Tallima H, Abou El Dahab M, El Ridi R. Role of T lymphocytes and papain enzymatic activity in the protection induced by the cysteine protease against Schistosoma mansoni in mice. J Adv Res. 2019;17:73-84. doi: 10.1016/j.jare.2018.12.008.

23. Dalton JP, Smith AM, Clough KA, Brindley PJ. Digestion of haemoglobin by schistosomes: 35 years on. Parasitol Today 1995;11(8):299-303.

24. Delgado VS, McLaren DJ. Schistosoma mansoni: evidence that sitedependent host responses determine when and where vaccine immunity is expressed in different rodent species. Parasitology 1990;100 Pt 1:57-63.

25. El Ridi R, Tallima H. Physiological functions and pathogenic potential of uric acid: A review. J Adv Res. 2017;8(5):487-493. doi: 10.1016/j.jare.2017.03.003. 